\title{
The Leiden MEMS Tribometer: Real Time Dynamic Friction Loop Measurements With an On-Chip Tribometer
}

\author{
W. Merlijn van Spengen · Joost W. M. Frenken
}

Received: 11 May 2007 / Accepted: 30 July 2007/Published online: 14 August 2007

(C) Springer Science+Business Media, LLC 2007

\begin{abstract}
On-chip MEMS tribometer devices until now have been much less sophisticated for dynamically sensing frictional forces than their FFM (friction force microscope) counterparts. In this article, we present a MEMS-based tribometer that can be used to measure dynamically, onchip and in-situ, the frictional properties of MEMS-scale contact geometries. The device provides the first FFM-like friction loops with contacting MEMS sidewall surfaces. Depending on the normal load two regimes of operation are identified. At low and intermediate loads, the frictional behaviour reflects wear-less relative motion of the silicon oxide surfaces of the MEMS device and we observe repeatable, irregular stick-slip behaviour, related to the surface roughness. At very high loads, wear causes changes in the topography of the contacting surfaces.
\end{abstract}

Keywords MEMS devices · Friction · Adhesion

\section{Introduction}

MEMS (micro-electromechanical systems) technology holds the promise to revolutionize the microelectronics industry, but reliability problems currently hamper the application of the technology in many fields [1]. The failure mechanisms of the mechanical MEMS components are widely differing from those of their macroscopic

W. M. van Spengen $(\bowtie)$ J. W. M. Frenken

Kamerlingh Onnes Laboratory, Leiden University, P.O. Box

9504, Leiden 2300RA, The Netherlands

e-mail: spengen@physics.leidenuniv.nl

W. M. van Spengen

Falco Systems, Gelderlandplein 75L, Amsterdam 1082LV,

The Netherlands counterparts. Failure mechanisms like stiction, charging, creep, and contamination have been studied in some detail. But friction and wear limit in the lifetime of MEMS components so much that reliable, commercially available microscopic devices have until now not employed sliding surfaces [2]. Beautiful focused ion beam (FIB) cross-sections of micro-motor test vehicles show that wear of MEMS with sliding surfaces can indeed be severe [3].

With the state-of-the-art in MEMS technology, this wear can hardly be prevented. This reflects that our physical knowledge of friction and wear on the length scales involved in contacting MEMS surfaces is very incomplete. Common lubricants used in macroscopic systems (such as oil) cannot be applied, because the viscous drag of these substances is so large that it prevents high speeds on the micro-scale, if the devices work at all.

\section{MEMS Tribometers}

Over the last 20 years, the development of the FFM (friction force microscope), a scanning probe microscope with atomic resolution, sensitive to lateral forces [4-7], has triggered renewed interest in the fundamentals of friction, by providing force information on contacts with atomic dimensions. This is complementary to that of the surface forces apparatus (SFA), which features large, atomically flat contacts, with excellent control over the distance between the contacting surfaces [5]. In order to address the phenomena at play when microscopic MEMS surfaces slide over each other, a measurement method is required that measures friction for contacts that are significantly larger than those in a typical FFM experiment, but with surfaces that are considerably less smooth than those in the SFA. In the ideal experiment from a MEMS point of view, 
two real MEMS surfaces should slide over each other onchip.

Several MEMS devices have been developed that can be used to measure friction on-chip. The first authors to report such a device were Lim et al. [8], who used a comb drive with dimples on the bottom side to contact and slide over the layer below the comb drive. They measured the static friction coefficient by displacing the comb drive electrostatically, clamping it, and monitoring the holding voltage required to prevent it from sliding back under the influence of the force of the restoring springs. This approach was later also used by Komvopoulos [9], who further proposed a way to measure the dynamic coefficient of friction with this device, although no experimental results of the latter type have been published.

Almost at the same time as Lim et al. [8] and Mehregany et al. [10] used a side-drive wobble motor to obtain the magnitude of the friction force by monitoring the actuation voltage required for starting the motion of the motor. Miller et al. [11] also used a micromotor to obtain friction data, in their case a micromotor with orthogonally oriented comb drive actuators. From a model of the motion of the motor under the influence of electrical stimuli, the dynamic coefficient of friction can be calculated. De Boer et al. [12, 13] developed a dedicated friction measurement device that uses a cantilever beam bent by electrostatic actuation, causing a hinged pad to slide over a counter-surface. By monitoring the bending of the cantilever at a certain applied actuation voltage with an optical interferometer, they could measure the static coefficient of friction as a function of normal load. Patton et al. used a lateral output motor to study friction between different structural layers [14].

The first to measure dynamic friction on sidewall surfaces only (important for, e.g. gears) were Prasad et al. [15] and Senft and Dugger [16]. The latter authors used a geometry that closely resembles the one reported in this article, but they used an optical readout technique to obtain the dynamic friction coefficient. With this device it was shown that SAM-coatings (self-assembled monolayer) significantly reduce friction and a $\mathrm{SiC}$ coating prevents wear [17-20]. Tas et al. [21] performed sidewall friction measurements with a more or less similar device.

The most recent example of a MEMS tribometer in the literature is the "Nanotractor", developed by De Boer et al. [22-27]. The nanotractor is a micromachined "inchworm actuator" that can walk over the surface in small steps against a restoring spring. It can also be used to obtain static friction coefficients as a function of normal load. Interestingly, it was found that small jumps can occur before the sliding starts. This effect was called "pre-sliding tangential deflections" [23]. The device was used in comprehensive tests with different coating materials [27].
Williams and Le [28] recently reviewed the methods to measure friction with MEMS devices.

MEMS tribometers do not only provide us with the possibility to do real-life friction tests with MEMS surfaces, but they also represent a unique opportunity to learn more about fundamental friction issues. FFMs always work under single- or few-asperity contact conditions. For that case, many interesting nano-tribological effects have been found, such as the reduction of friction through thermal activation (thermolubricity) and the nearly complete vanishing of friction among incommensurate atomic lattices (superlubricity) [29-31]. On the an other hand, results with macroscopic friction testers have never explicitly hinted to such effects. This means that somewhere in the transition from a low-force single-asperity contact to a high-force many-asperity contact, these peculiar types of behaviour are lost.

MEMS devices are unique in the sense that they have geometries and forces precisely in the range where this transition takes place. Depending on the normal load, which can vary from the same levels at which an AFM/ FFM typically operates to many micro-Newtons, the contact can contain only on or a few contacting asperities or many hundreds. A sliding contact can be either wear-less or exhibit considerable wear. The roughness is of the order over which the contact forces act, and the forces that are present naturally (surface forces) or that can be generated artificially (electrostatic actuation) also span a very important and interesting range. This makes MEMS an ideal technology to expand our fundamental knowledge of friction. In addition, research with MEMS-based tribometers holds the promise of understanding friction on this scale in more detail and hence reducing and eventually solving the friction and wear problems that currently hamper the technology so much.

Until now, MEMS tribometers for dynamic sensing of frictional forces have been much less sophisticated than their FFM counterparts. In this article, we present a MEMS-based tribometer that can be used to measure dynamicaly, on-chip and in-situ and the frictional properties of MEMS-scale contact geometries. This device has enabled us to measure the first FFM-like friction loops with contacting MEMS surfaces.

\section{Device}

The MEMS tribometer that we have developed (processed at MEMSCAP $^{1}$ in the PolyMUMPS process) resembles those of Senft and Dugger [16] and Tas et al. [21], with

\footnotetext{
${ }^{1}$ MEMSCAP Inc., 4021 Stirrup Creek Drive, Suite 120, Durham, NC, USA
} 
two orthogonally oriented comb drives that come together in a single point. The main difference is that in the device of Senft and Dugger the normal force is generated by pulling one of the orthogonal arms against a counter-surface, while in our case and that of Tas et al., this is achieved by pushing a slider, which can have various shapes, against a counter-surface opposite to it (Fig. 1a and b). The slider and the counter-surface are at DC ground potential.

One comb drive is used to push the slider against the counter-surface with a known force, while the other is used to exert a lateral force on the slider, along the countersurface. A calibration of the measured capacitance change due to the comb drive motion in $\mu \mathrm{m}$ can be obtained, because from the geometry we know that after a motion of $2.0 \mu \mathrm{m}$ the slider touches the counter-surface, which is clearly observable.

Instead of the optical detection used by Senft and Dugger [16] and Tas et al. [21], we use a fast, sensitive electronic readout system to monitor the position of actuated comb drives with high accuracy. In order to obtain accurate position readings, we have developed a method to obtain the capacitance changes of moving comb drives with high resolution in the presence of large, non-constant parasitic on-chip and cable capacitance [32]. The comb drive motion is intended to be fully in plane, but in practice it also has a small out-of plane component (levitation, first discussed by Tang et al. [33]). This out-of-plane motion influences the capacitance readings and also causes the device to move upward significantly as well as sideways, at low actuation voltages. We have developed an analytical model to extract from the motion-related capacitance change both the in-plane and out-of-plane component [34].

Using both techniques together, we have recently shown that it is possible to perform very accurate stiction force measurements with a 1-dimensional version of the Leiden MEMS Tribometer device, which we have called the "nano-battering ram" [35]. Here, we use the same electronic system to monitor the position of the comb drives of the MEMS Tribometer while applying both the normal force and the lateral force with high accuracy.

\section{Method to Measure Friction Loops}

By applying a voltage to the normal load comb drive, we here bring the slider in contact with the counter-surface (Fig. 2). For the experiments discussed in the next sections we have used the slider geometry shown in Fig. 3c. In the position reading (the capacitance measurement corrected for levitation effects), we see that the slider does not move any further once it touches the counter-surface (Fig. 4). Since it is known from the design that the restoring springs have a combined spring coefficient of approximately $1.3 \mathrm{~N} / \mathrm{m}$, the normal force can be calculated from the voltage that is used in excess of the value required to bring the slider into contact. In the retraction curve, we can see that the slider remains stuck down to a lower-holding voltage, which can calculate that the stiction force between the sidewalls of the slider and the counter-surface is $52 \mathrm{nN}$ at $27^{\circ} \mathrm{C}$ and $25 \% \mathrm{RH}$ (relative humidity).

Proper actuation of the device in the lateral direction, by applying actuation voltages to the lateral force comb drive, requires some extra attention. The modelling of the levitation effect (see Sect. 3 and ref. [34]) shows that at low voltages significant out-of plane motion occurs, saturating at an equilibrium height approximately $0.5 \mu \mathrm{m}$ above the rest position. By not operating the device around a lateral actuation voltage of $0 \mathrm{~V}$, but working in the offset window of $40-80 \mathrm{~V}$, where the levitation has already reached its saturation height, the out-of-plane motion cannot influence the measurement anymore. Of course, the levitation reduces the apparent contact area by $25 \%(2.0 \mu \mathrm{m}$ sidewall height). Typical lateral position measurements with and without contact are shown in Fig. 5. Since the capacitance changes are very small-the total change (not the resolution!) is around $5 \mathrm{fF}-1000$ averages have been used to
Fig. 1 (a) SEM micrograph of the Leiden MEMS Tribometer. (b) Schematic of the Leiden MEMS Tribometer
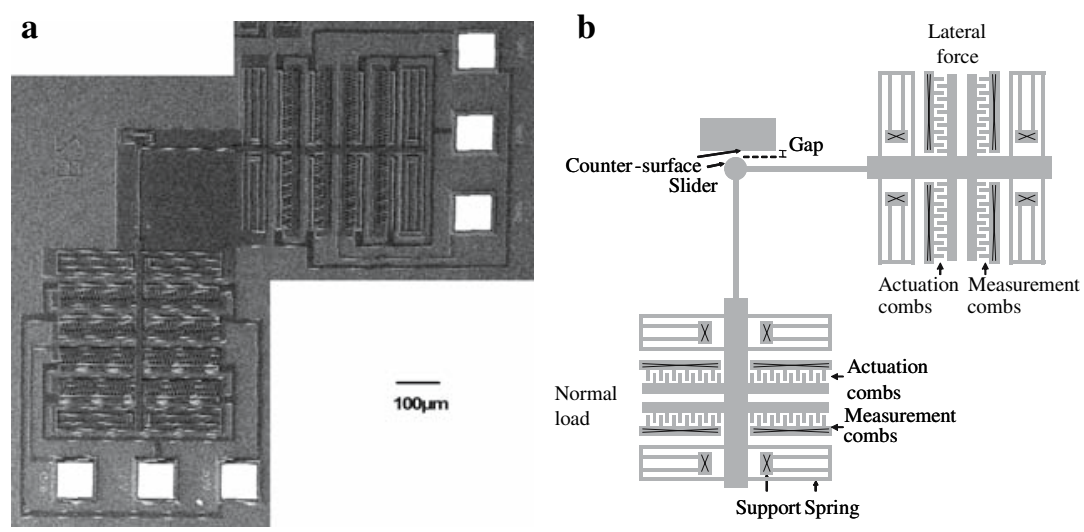

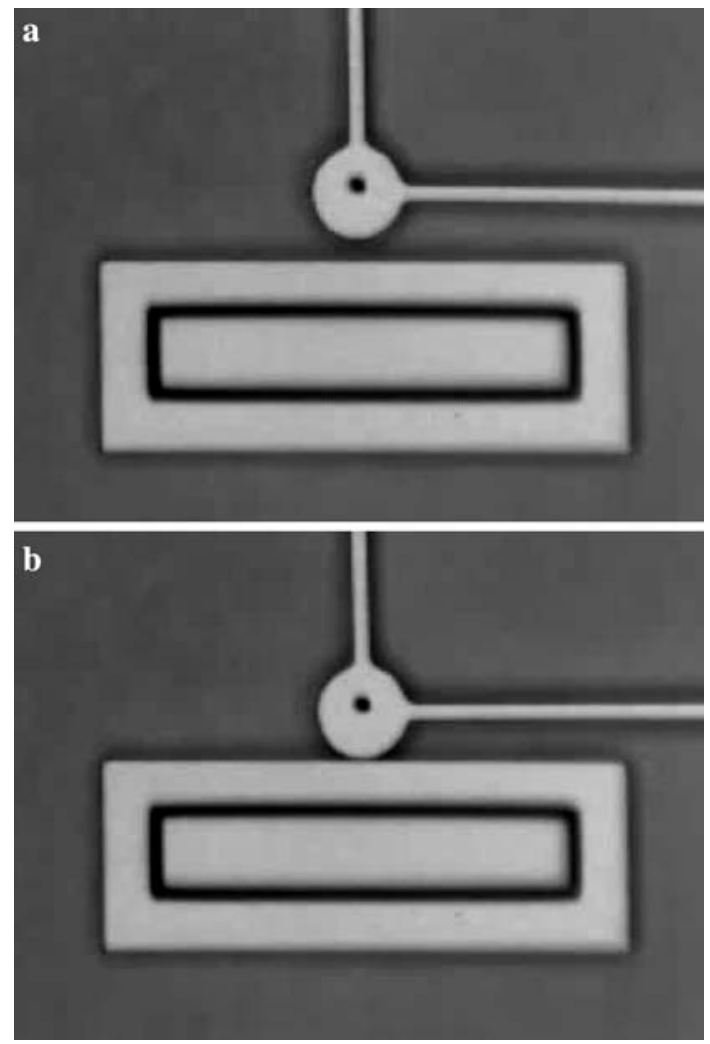

Fig. 2 Actuation of the Leiden MEMS Tribometer. A close up under an optical microscope of the touching surfaces of the friction version of the nano-battering ram [35]. Fig. (a) shows the moving slider (disk shaped) in its rest position, Fig. (b) shows it actuated and touching the counter-surface, immediately prior to a friction measurement. The slider is $20 \mu \mathrm{m}$ in diameter

obtain each of these curves, every forward and reverse run together taking $1 \mathrm{~s}$.

In order to convert our MEMS measurements to 'friction force loops', the common representation of FFM measurements, we have to introduce a "support position" for the horizontal axis and plot the lateral or friction force along the vertical axis. In FFM experiments, this "support position" is the position of the cantilever base, while the tip is lagging behind and twisting the cantilever, generating the FFM position signal. In the Leiden MEMS Tribometer, the whole device lags behind with respect to the actuation voltage, so there is no real support position. Instead, the position where the MEMS Tribometer would have been in response to the lateral actuation voltage in the absence of interaction with the counter-surface (dashed curve in Fig. 5) serves as the equivalent of the "support position". The lateral force is obtained by multiplying the distance by which the tribometer as a whole lags behind (difference between the solid and dashed curves in Fig. 5) with the combined lateral spring constant of the springs that generate the restoring force. Figure 6 shows the result of re-plotting the two measurements of Fig. 5 as friction loops.
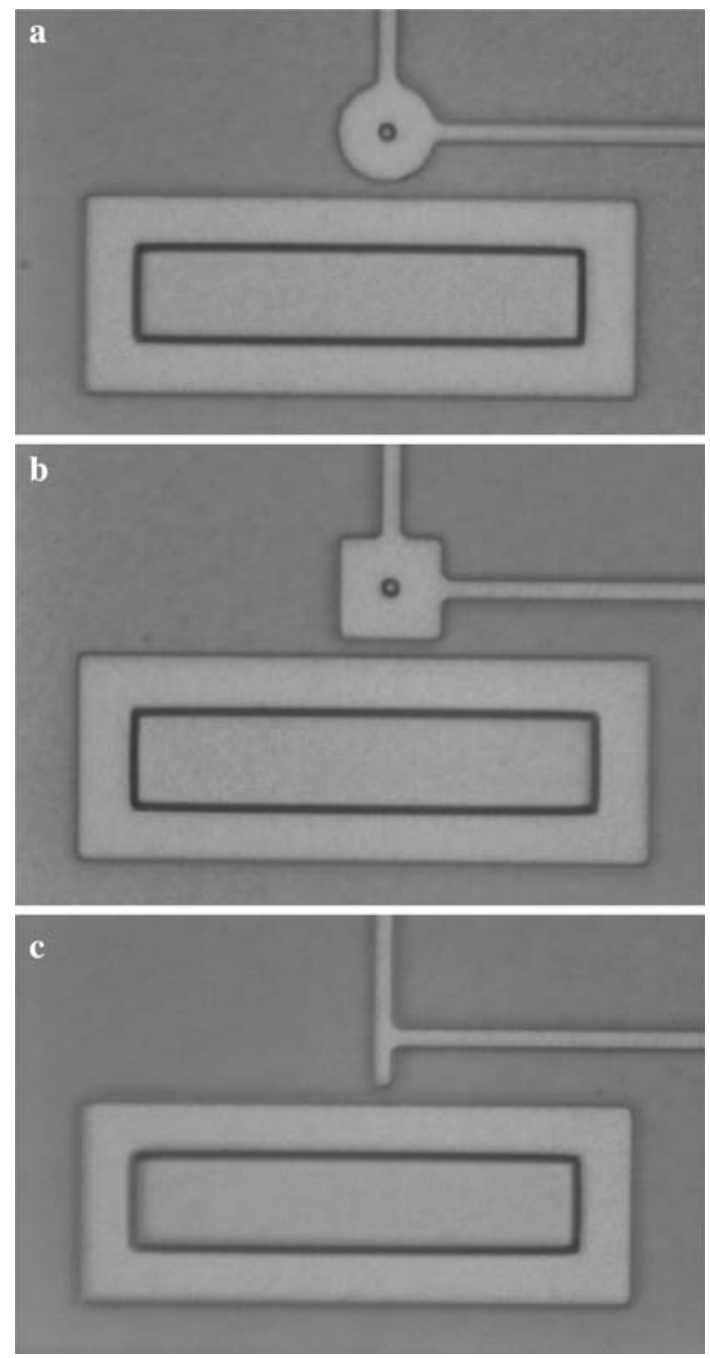

Fig. 3 Different sliders can be used for the friction experiments. The slider used for the experiments shown in this article is the "end of a beam" type slider (c)

The friction loop at non-zero normal force in Fig. 6 demonstrates that the device moves in a stick-slip fashion, where the tribometer slips between positions at which it is temporarily stuck. As mentioned above, the curves in Figs. 5 and 6 are the result of 1000 averages. The fact that the stick-slip character has not been averaged out over 1000 loops implies that the locations of the slip points are (more or less) repeatable in successive passages, which strongly suggests that during each trajectory the tribometer device gets stuck on the same elements in the surface topography (roughness) of the counter-surface.

\section{Friction Loops as a Function of Normal Load}

In Fig. 7, we show friction loops obtained for different normal forces. The experiments were performed, starting 


\section{Normal force on the contact $(\mathrm{nN})$}

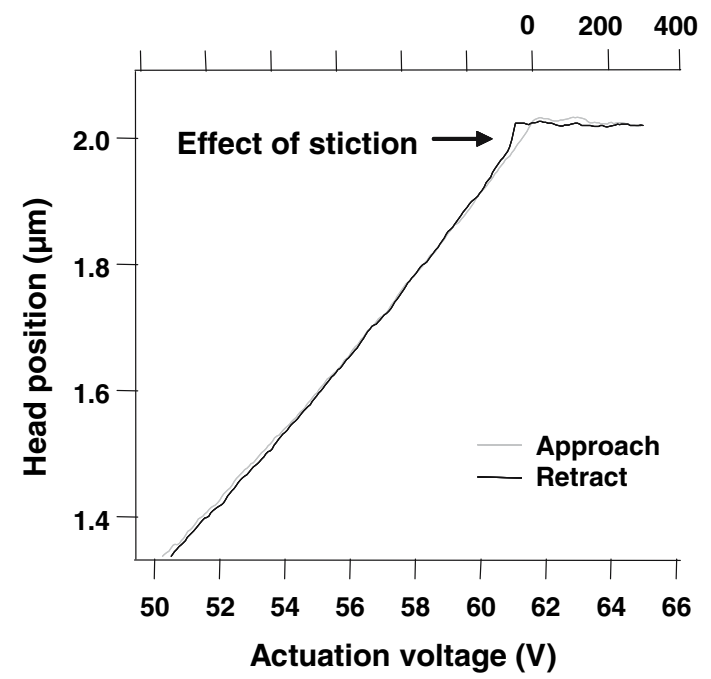

Fig. 4 Approach and retraction data of the normal force comb drive showing the result of the stiction force between the surfaces. From the distance jumped of $40 \mathrm{~nm}$ and the spring coefficient of $1.3 \mathrm{~N} / \mathrm{m}$, computed from the design, the stiction force can be calculated to be $52 \mathrm{nN}$

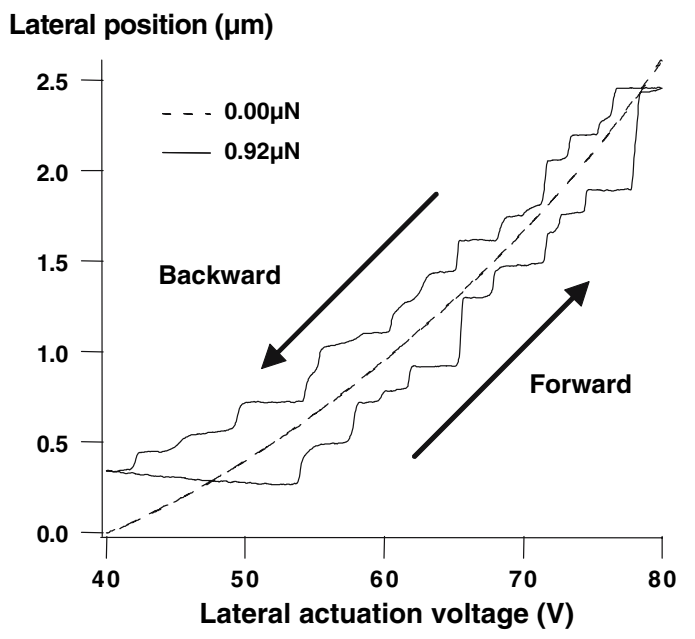

Fig. 5 Measured lateral slider position as a function of voltage on the lateral force comb drive for two settings of the normal force comb: (dashed curve) just out of contact and (solid curve) with $0.92 \mu \mathrm{N}$ of normal force on the contact

with a completely new sensor at the lowest normal load, so that in the lower-load measurements the two surfaces were not yet altered by possible wear during the higher-load measurements.

The friction loops in Fig. 7 correspond to two different regimes. Up to a normal load of $0.92 \mu \mathrm{N}$, stick-slip motion is observed that is reproduced over at least 1000 repetitions. At the even higher load of $1.42 \mu \mathrm{N}$, the stick-slip motion is much less pronounced. We attribute this higherload regime to wear. This interpretation is corroborated by

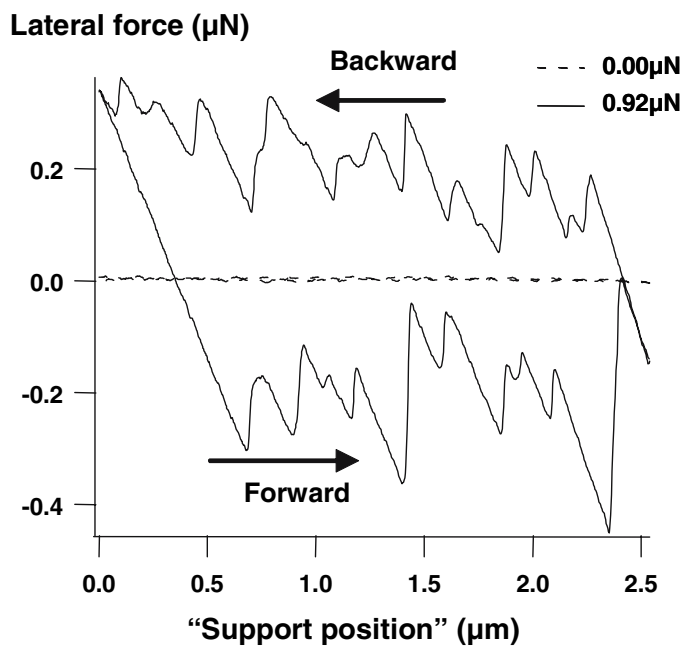

Fig. 6 High-resolution MEMS friction loops constructed using the measurements of Fig. 5: (dashed curve) just out of contact and (solid curve) with $0.92 \mu \mathrm{N}$ of normal force on the contact. The area enclosed by the loop is the energy dissipated by the friction process during one forward-reverse cycle

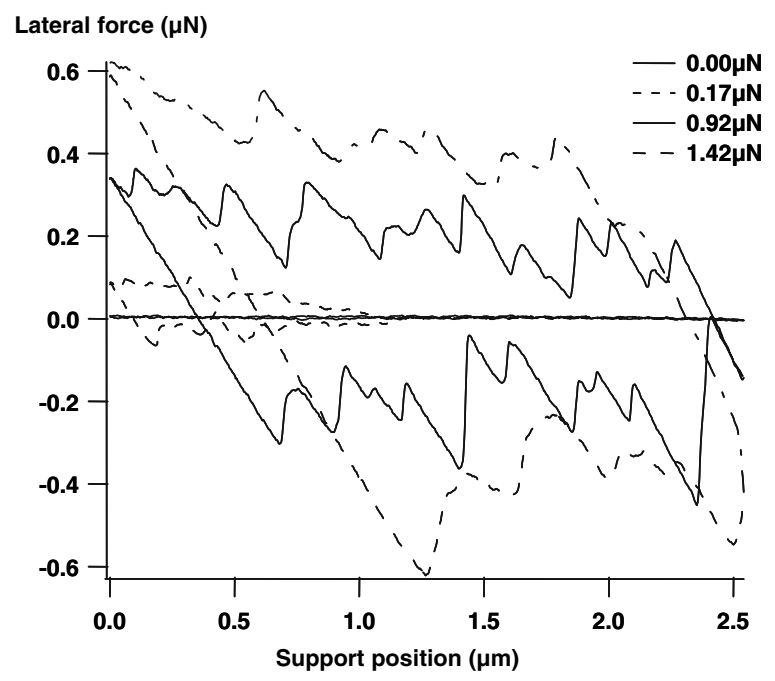

Fig. 7 Friction loops obtained by the Leiden MEMS Tribometer at different normal loads, starting with a pristine device and measured from lower to higher loads

the substantial difference in specific shape and overall character between measurements at the lower load of $0.92 \mu \mathrm{N}$, performed immediately before and after the highload experiment (Fig. 8), which shows that the higher-load changes have been irreversible. The removal of the stickslip motion suggests that during the higher-load sliding the sharpest protrusions on the counter-surface and probably also on the slider have been worn off.

In Fig. 7 we see that for the curve with a low normal load of $0.17 \mu \mathrm{N}$, the tribometer looses contact at a support position of approximately $1.3 \mu \mathrm{m}$. The reason is that the applied normal load is not constant along the trajectory. As 


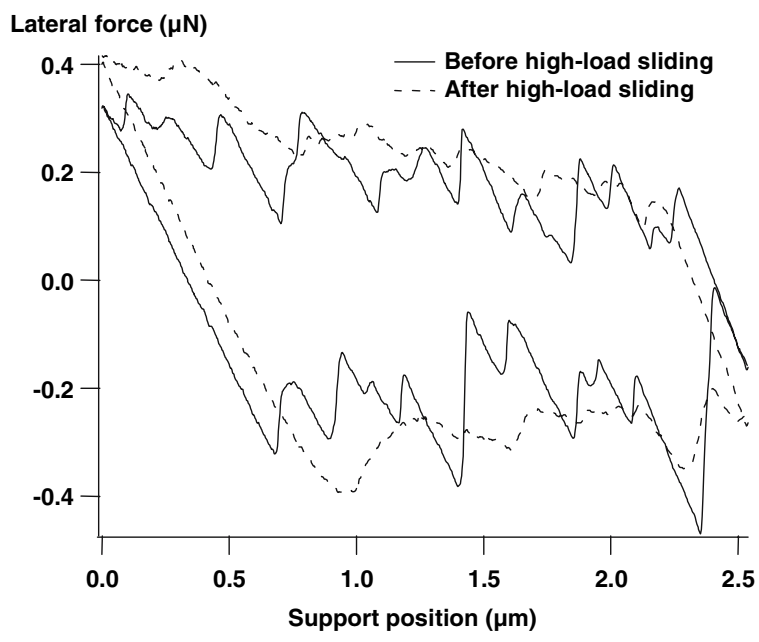

Fig. 8 Friction loops measured with the Leiden MEMS Tribometer at a normal load of $0.92 \mu \mathrm{N}$ immediately before (solid curve) and immediately after (dashed curve) a measurement at a high normal load of $1.42 \mu \mathrm{N}$. The irreversible change between the two measurements shows that the surface has undergone plastic deformation

is indicated in Fig. 9a, the free slider, not in contact with the counter-surface, would move in a circle-like trajectory, rather than to translate perfectly parallel to the countersurface. As a result, the normal force between slider and counter-surface decreases when the "support position" is increased, until the surfaces separate altogether. This is confirmed by the measurement in Fig. 9b, which shows that at a higher-lateral actuation voltage, contact is established at a higher-normal actuation voltage.

While the variation of normal load with lateral position might be regarded as an imperfection in the measurements, it provides an excellent opportunity to investigate the friction force as a function of normal load at low-load conditions (Fig. 10). The result shown here is very interesting. A significant lateral force is present even though immediately before separation the slider is actually subjected to a significant pulling force $F_{\text {pull }}$, which is directed away from the surface and close to cancelling the adhesion force $F_{a d h}$. In FFM experiments it has also been observed

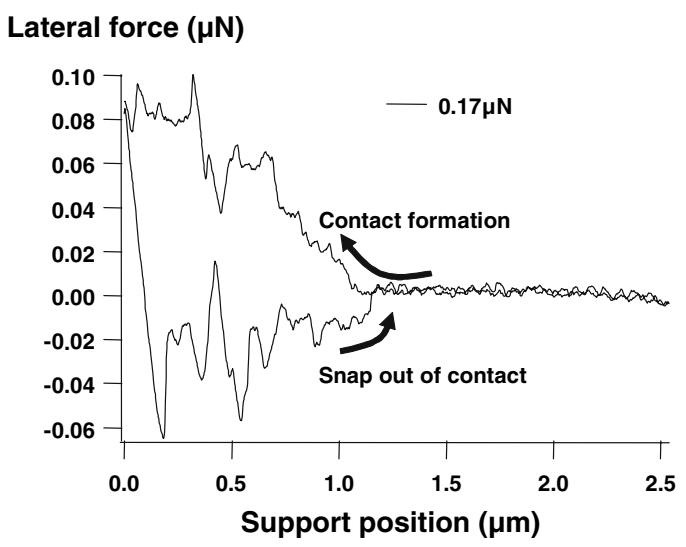

Fig. 10 Magnified view of the low-load data of Fig. 7, obtained at a normal force of $0.17 \mu \mathrm{N}$. We see that friction disappears when the slider snaps loose from the surface, and that friction builds up again when it returns into contact

that friction is still present when the loading force on the tip is reduced to zero or even made negative and the precise functional dependence has been employed to test different theoretical descriptions of the adhesion effect in a singleasperity contact [36].

\section{Outlook}

An important limitation in the present study is the fact that all the measurements shown are averages over 1000 subsequent, individual friction loops. This has been necessary in the current set up to obtain reasonable signal-to-noise figures. When the motion and the forces are fully reproducible from one friction loop to the next, this averaged representation of the data is justified. However, when subsequent friction loops are different, for example because wear is modifying the surface from one loop to the next, important information is washed out. This is illustrated in Fig. 11, which shows a 2-loop average and a 1000-loop average, both measured in the high-load regime, in which the surface is irreversibly changing. As expected,
Fig. 9 (a) Under the influence of lateral actuation, the slider of the Leiden MEMS tribometer would move in a circle-like trajectory, when it would not be in contact with the countersurface. (b) Measurements of the normal displacement versus normal actuation voltage for different values of the lateral actuation voltage confirm that a higher normal load voltage is required to make contact at higher lateral displacements a

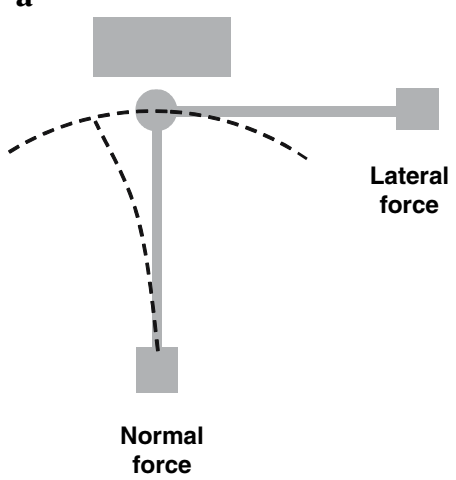

b

Normal load voltage required for contact $(\mathrm{V})$

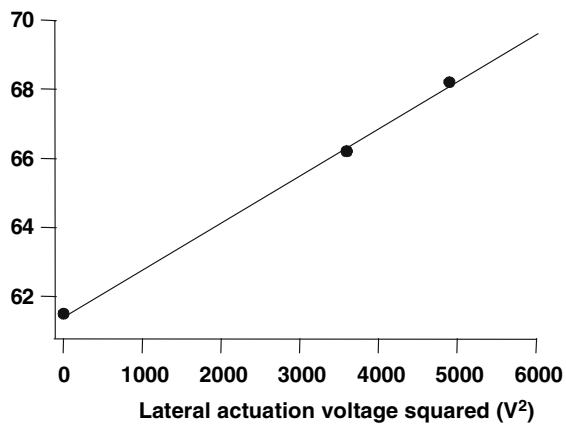




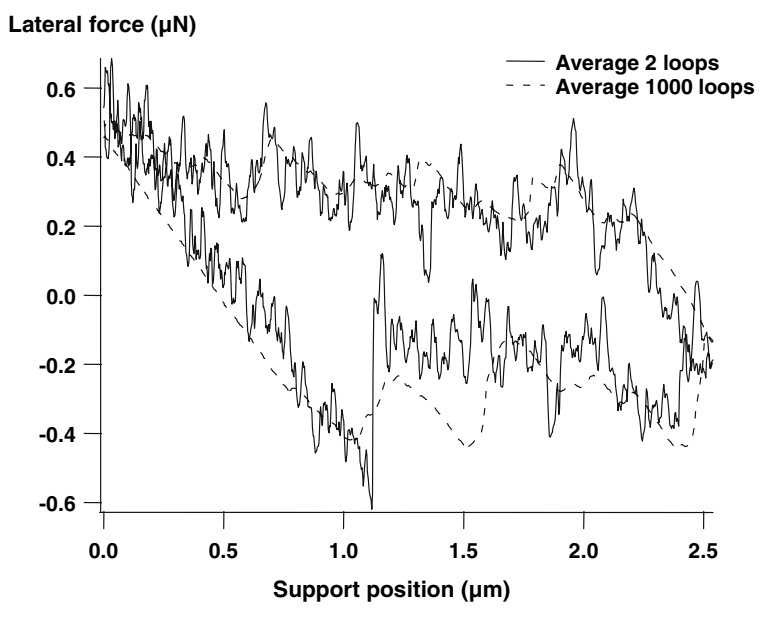

Fig. 11 Graph with the averages of 2 and 1000 individual friction loops, measured at a normal load of $1.16 \mu \mathrm{N}$. Irregular, irreproducible slip events that stand out in the noisy 2-loop average are smeared out and lost in the 1000-loop average

the 2-loop average is much more noisy, but in addition, we also observe several stick-slip events that are washed out completely or nearly completely in the 1000-loop average. For example, the 2-loop curve shows a significant slip event in the forward branch at a position of $1.15 \mu \mathrm{m}$. Only a smeared out force drop is present in the 1000-loop average, indicating that a slip event has occurred in most of the individual loops, but each time at a somewhat different location, reflecting the ongoing surface modification. Most of the other, less pronounced force variations of the 2-loop curve cannot be recognized at all in the 1000-loop average. This suggests that also the smooth measurements in Fig. 10 consist of many jumps at irregular positions and times that are smeared out by the averaging procedure.

We are currently improving our readout electronics to the point where we can clearly observe the detailed force variations within single loops. This will allow us to perform a meaningful investigation of individual jumps and to study the statistics of the friction and wear process. Another area that we are presently paying attention to is the calibration procedure for the forces. Rather than to rely on calculated spring constants, based on the designed dimensions of the device, we plan to determine the precise displacement-voltage characteristics of future MEMS devices using dedicated calibration structures.

Now that we have both the MEMS vehicle to do on-chip friction measurements dynamically with high resolution and the electronic means to read out the data with high resolution, we are set to explore the link between nanoscale and macroscopic friction. Many nano-tribological principles have been predicted, and to some extent studied with AFM/FFM. Examples are super- and thermolubricity [2931], the effect of solid, liquid and vapour phase lubricants on this scale [37], and the effect of different environmental conditions (e.g., humidity and temperature). Other future directions involve the effect of speed, contact type and the exact role of adhesion (stiction) and surface contamination on the nano and micro scale. This is important both for MEMS technology and from a more fundamental, physical point of view, because all common real contacts in everyday life are multi-asperity, contaminated, partly lubricated systems.

\section{Conclusions}

We have developed a MEMS device (the Leiden MEMS Tribometer) and an electronic measurement set up dedicated to sidewall surface friction experiments with two sliding MEMS surfaces. This has provided us with the unique opportunity to study dynamically, in situ and onchip the friction behaviour of contacting, sliding MEMS surfaces under realistic conditions. It is the first MEMS device that can be used to measure friction loops similar to those obtained with friction force microscopy.

With the Leiden MEMS Tribometer, we have identified two normal load regimes in the friction behaviour. At low loads, stick-slip motion is observed, which is repeatable over many runs, but is stochastic in nature as far as the stick/slip positions are concerned. We associate this regime with the wear-less sliding of the hard silicon oxide surfaces over one another. The stick-slip positions are presumably related to the surface roughness of the MEMS sidewalls. At high loads, the regular stick-slip disappears, and the surface is modified (wear), which is proven by the fact that a lower normal load measurement afterwards does not yield the same result as before the wear experiment. The fact that in the current high-load measurements, which are all 1000loop averages, no stick-slip features have been observed does not necessarily mean that the motion is a smooth sliding of the surfaces over one another. We have shown that stick-slip events still occur at high loads but that the stick-slip positions change over time due to the ongoing wear process.

With further improvements, our new Leiden MEMS Tribometer system will enable us to investigate several recently discovered nano-tribological effects with real MEMS surfaces under realistic working conditions.

Acknowledgements This work is financially supported by the Dutch NWO-STW foundation in the "Veni" program under ref no. LMF.7302.

\section{References}

1. Miller, W.M., Tanner, D.M., Miller, S.L., Peterson, K.A.: MEMS reliability: the challenge and the promise, 4th Annual "The Reliability Challenge”, Dublin, Ireland, p. 4.1. (1998) 
2. van Spengen, W.M.: MEMS reliability from a failure mechanisms perspective. Microelectron. Reliab. 43, 1049 (2003)

3. Tanner, D.M., Miller, W.M., Eaton, W.P., Irwin, L.W., Peterson, K.A., Dugger, M.T., Senft, D.C., Smith, N.F., Tanyunyong, P., Miller, S.L.: The effect of frequency on the lifetime of a surface micromachined microengine driving a load. International Reliability Physics Symposium Proceedings (IRPS) (1998) 26

4. Mate, C.M., McClelland, G.M., Erlandsson, R., Chiang, S.: Atomic scale friction of a tungsten tip on a graphite surface. Phys. Rev. Lett. 59, 1942 (1987)

5. Bhushan, B. (ed.): Springer handbook of nano-technology. 2nd edn. Springer, Berlin (2007) chapters 29-45

6. Carpick, R.W., Salmeron, M.: Scratching the surface: fundamental investigations of tribology with atomic force microscopy. Chem. Rev. 97, 1163 (1997)

7. Dienwiebel, M., de Kuyper, E., Crama, L., Frenken, J.W.M., Heimberg, J.A., Spaanderman, D.-J., Glastra van Loon, D., Zijlstra, T., van der Drift, E.: Design and performance of a highresolution frictional force microscope with quantitative threedimensional force sensitivity. Rev. Sci. Instrum. 76, 043704 (2005)

8. Lim, M.G., Chang, J.C., Schultz, D.P., Howe, R.T., White, R.M.: Polysilicon microstructures to characterize static friction. In: Proc. IEEE Workshop on Micro Electro Mechanical Systems, 11-14 February, Napa valley, CA, USA, p. 82. (1990)

9. Komvopoulos, K.: Surface engineering and microtribology for microelectromechanical systems. Wear 200, 305 (1996)

10. Mehregany, M., Senturia, S.D. and Lang, J.H.: Friction and wear in microfabricated harmonic side-drive motors, In: Proc. IEEE Solid-state Sensor and Actuator Workshop, p. 17 (1990).

11. Miller, S.L., Sniegowski, J.J., LaVigne, G., McWorther, P.J.: Friction in surface micromachined microengines. Proc. SPIE 2722, 197 (1996)

12. de Boer, M.P., Redmond, J.M., Michalske, T.A.: A hinged-pad test structure for sliding friction measurement in micromaching. Proc. SPIE 3512, 241 (1998)

13. de Boer, M.P., Mayer, T.M.: Tribology of MEMS. MRS Bull. 26 $302(2001)$

14. Patton, S.T., Cowan, W.D., Eapen, K.C., Zabinski, J.S.: Effect of surface chemistry on the tribological performance of a MEMS electrostatic lateral output motor. Trib. Lett. 9(3-4), 199 (2000)

15. Prasad, R., MacDonald, N., Taylor, D.: Micro-instrumentation for tribological measurement, In: Proc. International Conference on Solid-State Sensors and Actuators TRANSDUCERS '95, Stockholm, Sweden, p. 52. (1995)

16. Senft, D.C., Dugger, M.T.: Friction and wear in surface micromachined tribological test devices. Proc. SPIE 3224, 31 (1997)

17. Srinivasan, U., Houston, M.R., Howe, R.T., Maboudian, R.: Alkyltrichlorosilane-based self-assembled monolayer films for stiction reduction in silicon micromachines. J. Micromech. S. 7(2), 252 (1998)

18. Ashurst, W.R., Yau, C., Carraro, C., Maboudian, R., Dugger, M.T.: Dicholorosimethylsilane as an anti-stiction monolayer for MEMS: a comparison to the octadecyltrichlorosilane selfassembled monolayer. J. Micromech. Syst. 10(1), 41 (2001)

19. Romig, A.D., Dugger, M.T., McWorther, P.J.: Materials issues in microelectromechanical devices: science, engineering, manufacturability and reliability. A. mater. 51, 5837 (2003)

20. Ashurst, W.R., Wijesundra, M.B.J., Carraro, C., Maboudian, R.: Tribological impact of $\mathrm{SiC}$ encapsulation of released polycrystalline silicon microstructures. Trib. Lett. 17(2), 195 (2004)
21. Tas, N.R., Gui, C., Elwenspoek, M.: Static friction in elastic adhesion contacts in MEMS. J. Adh. Sci. Technol. 17(4), 547 (2003)

22. de Boer, M.P., Luck, D.L., Ashurst, W.R., Maboudian, R., Corwin, A.D., Walraven, J.A., Redmond, J.M.: High-performance surface-micromachined inchworm actuator. J. Micromech. Sys. 13(1), 63 (2004)

23. Corwin, A.D., Street, M.D., Carpick, R.W., Ashurst, W.R. de Boer, M. P.: Pre-sliding tangential deflections can govern the friction of MEMS devices. In: Proc. TRIB2004, the ASME/STLE Internation Joint Tribology Conference, Long beach, CA, USA, p. 1. (2004)

24. Corwin, A.D., de Boer, M.P.: Effect of adhesion on dynamic and static friction in surface micromachining. Appl. Phys. Lett. 84(13), 2451 (2004)

25. Starr, M.J., Reedy, E.D., Corwin, A.D., Carpick, R.W. Flater, E.E.: Contact mechanics description of inelastic displacement of a nano-positioning device, In: Proc. ICMENS'05, IEEE International Conference on MEMS, NANO and Smart Systems, p. 421. (2005)

26. Flater, E.E., Corwin, A.D., de Boer, M.P., Carpick, R.W.: In situ wear studies of surface micromachined interfaces subject to controlled loading. Wear 260, 580 (2006)

27. Corwin, A.D., Street, M.D., Carpick, R.W., Ashurst, W.R., Starr, M.J. de Boer, M.P.: Friction of different monolayer lubricants in MEMs interfaces, Sandia report SAND2005-7954, Sandia National Laboratories, Albuquerque, CA, USA, (2006)

28. Williams, J.A., Le, H.R.: Tribology and MEMS. J. Phys. D: Appl. Phys. 39, R201 (2006)

29. Dienwiebel, M., Verhoeven, G.S., Pradeep, N., Frenken, J.W.M., Heimberg, J.A., Zandbergen, H.W.: Superlubricity of graphite. Phys. Rev. Lett. 92(12), 126101-1 (2004)

30. Socoliuc, A., Bennewitz, R., Gnecco, E., Meyer, E.: Transition from stick-slip to continuous sliding in atomic friction: entering a new regime of ultralow friction. Phys. Rev. lett. 92(13), 1343011 (2004)

31. Krylov S.Yu., Jinesh, K.B., Valk H., Dienwiebel, M., Frenken, J.W.M.: Thermally induced suppression of friction at the atomic scale. Phys. Rev. E71(6), 065101-1 (2005)

32. van Spengen, W.M., Oosterkamp, T.H.: A sensitive electronic capacitance measurement system to measure comb drive motion of surface micromachined MEMS devices. J. Micromech. Microeng. 17(4), 828 (2007)

33. Tang, W.C., Lim, M.G., Howe, R.T.: Electrostatic comb drive levitation and control method. J. Micromech. S. 1(4), 170 (1992)

34. van Spengen, W.M., Heeres, E.C.: A method to extract the lateral and normal component of the motion of an actuated MEMS comb drive from the capacitance change. J. Micromech. Microeng. 17, 447 (2007)

35. van Spengen, W.M., Bakker, E., Frenken, J.W.M.: A 'nano-battering ram' for measuring surface forces: obtaining forcedistance curves and sidewall stiction data with a MEMS device. J. Micromech. Microeng. 17(7), S91 (2007)

36. Enachescu, M., van den Oetelaar, R.J.A., Carpick, R.W., Ogletree, D.F., Flipse, C.F.J., Salmeron, M.: Atomic force microscopy study of an ideally hard contact: the diamond(111)/tungsten carbide interface. Phys. Rev. Lett. 81, 1877 (1998)

37. Strawhecker, K., Asay, D.B., McKinney, J., Kim, S.H.: Reduction of adhesion and friction of silicon oxide surface in the presence of $n$-propanol vapour in the gas phase. Tribol. Lett. 19(1), 17 (2005) 